\title{
Ictiofauna de campos alagados da Ilha do Marajó, Estado do Pará, Brasil
}

\author{
Luciano Fogaça de Assis Montag ${ }^{1,3}$, Adna Almeida de Albuquerque ${ }^{2}$, \\ Tiago Magalhães da Silva Freitas ${ }^{2} \&$ Ronaldo Borges Barthem ${ }^{2}$ \\ ${ }^{1}$ Laboratório de Ecologia e Zoologia de Vertebrados, Instituto de Ciências Biológicas, \\ Universidade Federal do Pará - UFPA, \\ CP 479, CEP 66075-110, Belém, PA, Brasil \\ ${ }^{2}$ Coordenação de Zoologia, Campus de Pesquisa, Museu Paraense Emílio Goeldi \\ Av. Perimetral, 1901, CP 399, Montese, Belém, PA, Brasil, www.museu-goeldi.br \\ ${ }^{3}$ Autor para correspondência: Luciano Fogaça de Assis Montag, e-mail: Ifamontag@gmail.com
}

MONTAG, L.F.A., ALBUQUERQUE, A.A., FREITAS, T.M.S. \& BARTHEM, R.B. The Ichthyofauna of Savannas from Marajó Island, State of Pará, Brazil. Biota Neotrop., 9(3): http://www.biotaneotropica.org. br/v9n3/en/abstract?inventory+bn01609032009.

\begin{abstract}
The flooded areas or savannas of the Marajó island is a fraction of the "Cerrado" biome in the Amazonian, and it is considered of a great importance to management plans, conservation and environmental preservation. In order to increase the knowledge of ichthyofauna from this region, the present study united information collected in other past campaigns, compiled from literature and selected from ichthyological collections on the fauna of the eco-region of Marajó's savanna. The field sampling occurred between the years of 2003 and 2005, and was applied the hand net, trawl net, gillnets, blockage and capture by free diving technique, which during this period were sampled the municipalities Ponta de Pedras, Chaves and Muaná. Besides these, four municipalities had also been included in past studies. The efficiency of this biological inventory was evaluated by species accumulation method (rarefaction) and the richness estimator Bootstrap. In these three places were collected 146 species, which 89 species $(60 \%)$ and eight families of Actinopterygii were included as new records for the island of Marajó. With the increase of collections in the municipalities of Ponta de Pedras, Chaves and Muaná, the total species of the island is 254 species. Of these, 44 species (17\%) are targets of commercial fishing for food and 46 species (18\%) are caught to ornamental business. Thus, biological inventories realized at places with a great environmental importance as the savannas or flooded areas of the Marajó Island are essential, because without this documentation, any measurement of biodiversity, delimitation of endemism areas and geographic distribution of species for implementation of conservation plans would be damaged. Therefore, it is necessary not only create conditions to protect wildlife, but to make it better known through mechanisms to financing inventory projects and taxonomy and systematic studies.
\end{abstract}

Keywords: fishes, biodiversity, savanna, Amazon basin.

MONTAG, L.F.A., ALBUQUERQUE, A.A., FREITAS, T.M.S. \& BARTHEM, R.B. Ictiofauna de campos alagados da Ilha do Marajó, Estado do Pará, Brasil. Biota Neotrop., 9(3): http://www.biotaneotropica.org. br/v9n3/en/abstract?inventory+bn01609032009.

Resumo: Os campos alagados ou savanas da ilha do Marajó é uma área disjunta do bioma Cerrado na Amazônia, e é considerada de grande importância para planos de manejo, conservação e preservação ambiental. A fim de ampliar o conhecimento ictiofaunístico da região, o presente estudo reuniu informações coletadas em campo, compiladas da literatura e selecionadas de coleções ictiológicas sobre a fauna de peixes da eco-região savana do Marajó. As coletas de campo ocorreram entre os anos de 2003 e 2005, e apresentaram como métodos a rede de mão, rede de arrasto, rede de tapagem e captura manual por técnica de mergulho, sendo que durante esse período foram amostrados os municípios de Ponta de Pedras, Chaves e Muaná. Além desses, outros quatro municípios também foram abordadas em estudos pretéritos. A eficiência desse inventário biológico foi avaliada pelos métodos de acumulação de espécies (rarefação) e pela estimativa de riqueza Bootstrap. Nestes três locais foram coligidas 146 espécies, sendo que destas, 89 espécies (60\%) e oito famílias da classe Actinopterygii foram registradas como novas ocorrências para os campos do Marajó. Com o incremento das coletas nos três municípios, o total de espécies para os campos da ilha é de 254 espécies. Desse total, 44 espécies (17\%) são alvos da pesca comercial para fins de alimentação e 46 espécies (18\%) são capturadas na pesca ornamental. Sendo assim, inventários biológicos realizados em locais de grande importância ambiental como ou campos alagados da Ilha do Marajó são imprescindíveis, pois sem esta documentação, qualquer avaliação de biodiversidade, delimitação de áreas de endemismo e distribuição geográfica para execução de medidas conservacionistas fica prejudicada. Torna-se necessário, portanto, não apenas criar condições para proteger a fauna, mas para torná-la melhor conhecida através da criação de mecanismos de apoio a projetos de inventariamento e estudos de taxonomia e sistemática.

Palavras-chave: peixes, biodiversidade, savana, Bacia Amazônica. 


\section{Introdução}

As "Savanas da ilha do Marajó" ou campos alagados foram classificados no Workshop 98, em Brasília, sobre Bioma Cerrado e Pantanal na categoria de alta importância biológica e recomendada prioritariamente à realização de inventários biológicos. Apesar de se situar na Amazônia, esta área foi tratada como sendo uma área disjunta do bioma Cerrado na Amazônia e considerada prioritária por apresentar uma fauna rica, com espécies endêmicas, e por estar sujeita a pressão antrópica, tanto pela expansão da agropecuária como pela construção de hidrovia (MPEG 2005).

Com o objetivo de atender esta demanda, o presente trabalho reuniu informações coletadas em campo, compiladas da literatura e selecionadas de coleções ictiológicas sobre a fauna de peixes da ecoregião do Marajó. As coletas de campo foram realizadas em três municípios, dos doze da ilha de Marajó, são eles: Ponta de Pedras, Muaná e Chaves. Estes municípios estão contidos na área demarcada pelos polígonos, que foram estabelecidos como áreas das manchas de savana, determinados pelo projeto Avaliação Ecológica e Seleção de Áreas Prioritárias à Conservação de Savanas Amazônicas, Arquipélago do Marajó, Estado do Pará. O presente artigo é resultado deste projeto, que é apoiado pelo Projeto de Conservação e Utilização Sustentável da Diversidade Biológica Brasileira (PROBIO), do Ministério do Meio Ambiente. Este visou reunir conhecimento científico sobre a biologia da eco-região savana do Marajó para subsidiar o estabelecimento de estratégias de i) gestão territorial, ii) zoneamento ecológico e sócio-ambiental, iii) manejo de recursos naturais e iv) conservação dos campos alagados do Marajó.

Uma das primeiras listas de espécies de peixes sobre o Marajó foi elaborada por Boulenger (1897), com base no material enviado pelo Dr. E. A. Goeldi, diretor na época do Museu do Pará, atual Museu Paraense Emílio Goeldi. Infelizmente, esta foi uma das poucas publicações sobre a ictiofauna desta região, sendo os campos ou savanas alagados do Marajó carentes de informações sobre ecologia e diversidade de sua biota aquática. Neste contexto, o presente trabalho visa contribuir no inventário das espécies de peixes da região das savanas do Marajó.

\section{Caracterização Geográfica da Região do Marajó}

A desembocadura dos Rios Amazonas e Tocantins é parcialmente bloqueada por um conjunto de arquipélagos que formam um delta interno na foz Amazônica. Os arquipélagos mais importantes são os de Bailique, Caviana, Marajó e o do Rio Pará. A ilha de Marajó possui $49.606 \mathrm{~km}^{2}$ de extensão e é a maior ilha deste complexo de arquipélagos, seguida por Caviana, Mexiana e Gurupá. (Sioli 1966, Miranda-Neto 1968; IBGE 1991).

A ilha do Marajó situa-se nas proximidades da linha do equador, que passa quase paralela ao norte da ilha. Suas coordenadas geográficas estão entre os paralelos $0^{\circ} 10^{\prime} \mathrm{S}$ a $1^{\circ} 48^{\prime} \mathrm{S}$ e $48^{\circ} 22^{\prime} \mathrm{O}$ a $50^{\circ} 49^{\prime} \mathrm{O}$. O noroeste da ilha é banhado pela foz do Rio Amazonas, onde ocorre uma série de outras linhas menores. Ao sul a ilha é separada do continente pelo Rio Pará que, a sudeste, expande-se recebendo as águas do rio Tocantins e outros rios menores, passando então a chamar-se de Baía do Marajó. Ao sudoeste, diversos canais ou furos limitam a ilha, enquanto a contra-costa, a noroeste é banhada pelo atlântico (Cruz 1987, Nascimento et al. 1991).

A ilha do Marajó pode ser dividida em duas regiões no sentido leste e oeste. Ao leste situam os campos alagados ou savanas amazônicas (Brasil 1999); que foi indicada como de alta prioridade no Workshop sobre os Biomas Cerrado e Pantanal em 1998. Esta paisagem domina praticamente toda a extensão dos municípios de Cachoeira do Ararí, Chaves, Soure, Salva Terra, Ponta de Pedras, Muaná e Santa Cruz do Ararí (Cruz 1987).

A região oeste da ilha apresenta um relevo mais elevado, com altitudes acima de $20 \mathrm{~m}$ em alguns pontos. Esta região cobre uma área de $26.560 \mathrm{~km}^{2}$, que pertence aos municípios de Afuá, Curralinho, São Sebastião da Boa vista, Breves e Anajás, e é caracterizada por possuir diversos canais, rios e pequenas ilhas, sendo por isto denominada de região dos furos (Cruz, 1987).

Os três municípios que se encontram nas machas de savanas definido pelo projeto “Avaliação Ecológica e Seleção de Áreas Prioritárias à Conservação de Savanas Amazônicas, Arquipélago do Marajó, Estado do Pará", são drenados por diversos rios que correm no seu interior e ao redor da ilha. Os municípios de Ponta de Pedras e Muaná localizam-se ao sul da ilha e é banhado pelo Rio Pará e o de Chaves localiza-se ao norte banhado pelas águas do Rio Amazonas. A sede municipal do município de Ponta de Pedras situa-se a $01^{\circ} 23$ ' $42^{\prime \prime} \mathrm{S}$ e $48^{\circ}$ 52' 18" O e é banhada pelo Rio Marajó-Açu, que deságua no Rio Pará. Além desse, outros rios importantes drenam o município, são eles: o Arari e seus afluentes, o Anabujú e Anajás. O principal curso d'água do município de Muaná é o Rio Pará, que correm em frente à sua sede municipal, localizada a $01^{\circ} 31^{\prime} 55^{\prime}$ ' S e $49^{\circ} 13^{\prime} 10^{\prime \prime} \mathrm{O}$.

$\mathrm{O}$ município de Chaves pertence à meso-região Marajó e à microrregião Arari, a sede municipal situa-se a $00^{\circ} 10^{\prime} 00^{\prime \prime} \mathrm{S}$ e $49^{\circ} 59^{\prime} 18^{\prime \prime}$ O. O principal rio é o Cururu, que corta o município de leste a oeste (SEPOF, 2007).

Tabela 1. Localidades de coletas nas manchas de savanas (polígonos) de Ponta de Pedras, Chaves e Muaná, Ilha do Marajó, Pará, Brasil.

Table 1. Sampling localities in savannas (polygons) of Ponta de Pedras, Chaves and Muaná, Marajó Island, Pará, Brazil.

\begin{tabular}{|c|c|c|c|}
\hline Localidades de Coleta & Corpo(s) d'água & Polígono & Coordenadas \\
\hline Igarapé Querepetuba & Igarapé & Ponta de Pedras & $01^{\circ} 22^{\prime} 08,1^{\prime \prime} \mathrm{S}$ e $48^{\circ} 58^{\prime} 20,8^{\prime \prime} \mathrm{O}$ \\
\hline Sítio Castanhal & Igarapé e várzea & Ponta de Pedras & $01^{\circ} 22^{\prime} 09,3^{\prime \prime} \mathrm{S}$ e $48^{\circ} 55^{\prime} 24,7^{\prime \prime} \mathrm{O}$ \\
\hline Fazedinha & Lago & Ponta de Pedras & $01^{\circ} 21^{\prime} 17,0^{\prime \prime} \mathrm{S}$ e $48^{\circ} 55^{\prime} 54,7^{\prime \prime} \mathrm{O}$ \\
\hline Mangaba & Lagos temporários & Ponta de Pedras & $01^{\circ} 26^{\prime} 40,2^{\prime \prime} \mathrm{S}$ e $49^{\circ} 00^{\prime} 30,0^{\prime \prime} \mathrm{O}$ \\
\hline Igarapé do baiano & Igarapé & Ponta de Pedras & $01^{\circ} 21^{\prime} 12,4^{\prime \prime} \mathrm{S}$ e $48^{\circ} 57^{\prime} 04,7^{\prime \prime} \mathrm{O}$ \\
\hline Igarapé Miri & Igarapé & Chaves & $00^{\circ} 11^{\prime} 57,5^{\prime \prime} \mathrm{S}$ e $49^{\circ} 59^{\prime} 37,6^{\prime \prime} \mathrm{O}$ \\
\hline Lago Arapapá & Lago temporário & Chaves & $00^{\circ} 12^{\prime} 18,7^{\prime \prime} \mathrm{S}$ e $49^{\circ} 59^{\prime} 01,8^{\prime \prime} \mathrm{O}$ \\
\hline Igarapé Tramaria & Igarapé & Chaves & $00^{\circ} 11^{\prime} 36,1^{\prime \prime} \mathrm{S}$ e $49^{\circ} 58^{\prime} 32,2^{\prime \prime} \mathrm{O}$ \\
\hline Igarapé Tamatatuba & Igarapé e lago de maré & Chaves & $00^{\circ} 11^{\prime} 20,2^{\prime \prime} \mathrm{S}$ e $49^{\circ} 53^{\prime} 12,5^{\prime \prime} \mathrm{O}$ \\
\hline Rio Atuá & Igarapé, várzea e lago de maré & Muaná & $01^{\circ} 16^{\prime} 06,9^{\prime \prime} \mathrm{S}$ e $49^{\circ} 23^{\prime} 02,2^{\prime \prime} \mathrm{O}$ \\
\hline Rio Tauá & Igarapé, várzea e lago de maré & Muaná & $01^{\circ} 17^{\prime} 00,6^{\prime \prime}$ S e $49^{\circ} 16^{\prime} 43,4^{\prime \prime} \mathrm{O}$ \\
\hline Igarapé Bom Jardim & Igarapé, várzea e lago de maré & Muaná & $01^{\circ} 16^{\prime} 19,6^{\prime \prime} \mathrm{S}$ e $49^{\circ} 23^{\prime} 38,5^{\prime \prime} \mathrm{O}$ \\
\hline Igarapé Jacarequa & Igarapé, várzea e lago de maré & Muaná & $01^{\circ} 15^{\prime} 38,5^{\prime \prime} \mathrm{S}$ e $49^{\circ} 19^{\prime} 07,4^{\prime \prime} \mathrm{O}$ \\
\hline
\end{tabular}




\section{Material e Métodos}

As informações analisadas neste trabalho foram obtidas de dados secundários e de coletas de campo realizadas entre os anos de 2003 a 2005. Os dados secundários foram obtidos dos portais eletrônicos, tais como Fishbase (www.fishbase.org) e SIBIP/NEODAT III (http:// www.mnrj.ufrj.br/search1c.htm) e da base de dados da coleção Ictiológica do Museu Paraense Emílio Goeldi. A confirmação do status taxonômico válido para as espécies foi baseada nos trabalhos de Reis et al. (2003) e Buckup et al. (2007).

\section{Coleta e análise de dados}

Entre os anos de 2003 e 2005 três expedições foram realizadas no âmbito do projeto do PROBIO Marajó (MPEG/MMA). Em dezembro de 2003 foi realizada uma expedição ao município de Ponta de Pedras, apresentando um maior esforço de coleta na bacia do rio Marajó-Açu. A segunda expedição foi realizada no município de Chaves no mês de agosto de 2004. E no mês de janeiro de 2005 a expedição foi realizada no município de Muaná, utilizando-se como sede, a fazenda Socorro em Berta Grande, a quatro horas do município (Tabela 1 e Figura 1).

A ictiofauna foi capturada nos três polígonos por meio de rede de mão (peneira) com um esforço de duas horas por coletor, rede de arrasto de fio e náilon com esforço de uma hora, rede de tapagem e captura manual por técnica de mergulho. Nos três locais de coleta foram estabelecidas 42 unidades de amostras.

Os espécimes coletados foram fixados em formol $10 \%$ e preservados em álcool $70 \%$. As identificações dos peixes foram baseadas em bibliografia especializada (Ellis 1913, Géry 1977, Isbrücker 1981, Burgess 1989, Buckup 1993, Mago-Leccia 1994, Reis 1997) e por comparações com coleções e apoio de especialistas. Após a identificação, os espécimes foram depositados na coleção do MPEG (Pará, Brasil) (anexo). A eficiência do inventário dos campos alagados do Marajó foi avaliada por métodos de acumulação de espécies (rarefação), pelo programa PAST ${ }^{\circledR}$ (Palaeontological Statistics, ver. 1.75) e estimativas de riqueza Bootstrap (Colwell \& Coddington, 1994) pelo programa Estimates ${ }^{\circledR}$ 8.0. com 1.000 aleatorizações. Esse último é um estimador de riqueza de dados de incidências que se baseia na

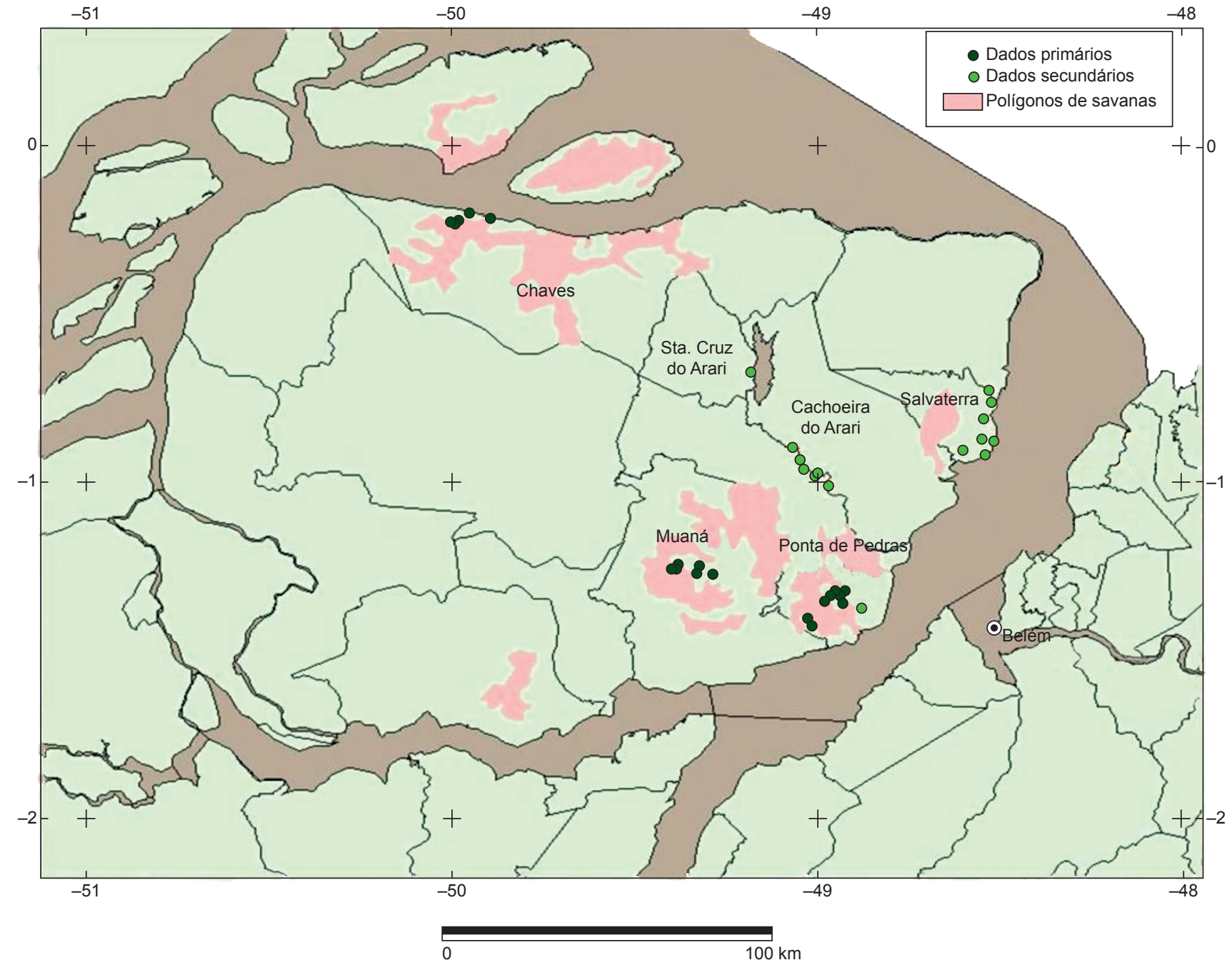

Figura 1. Distribuição dos dados primários (preto) e secundários (vermelho) na região de campos da Ilha do Marajó (Pará) entre os anos de 1905 a 2005.

Figure 1. Distribution of primary (black) and secondary data (red) in areas or savannas of the Marajó Island (Pará) between the years 1905 and 2005. 
proporção de espécies raras (coletadas apenas uma ou duas vezes nas amostras) para estimar a riqueza total de espécies e o desvio padrão (Colwell e Coddigton 1994, Colwell 1997). Foram estabelecidos como sendo a unidade de amostra os anos correspondentes a 1923 a 2005.

\section{Resultados e Discussão}

Até o ano de 1999 estavam registrados para o Marajó 818 registros, com 165 espécies em 37 famílias de peixes relatadas para as manchas de campos da Ilha do Marajó, o que representa aproximadamente 7\% da riqueza de peixes da Bacia Amazônica. Tais registros são na maioria de coleções ictiológicas nacionais, pertencentes ao Museu Paraense Emilio Goeldi - MPEG e Museu de Zoologia da Universidade do Estado de São Paulo - MZUSP e coleções internacionais, como o Museu de História Natural da Suécia (NMR) e Museu do Estado da Flórida (UF). Os registros da coleção ictiológica do Museu Paraense Emílio Goeldi totalizam 800 lotes de peixes. A maior parte do material desta coleção foi coletada entre as décadas de 80 e 90 e principalmente nos municípios de Cachoeira do Arari, Muaná, Ponta de Pedras, Salvaterra, Santa Cruz do Arari e Soure.

\section{Riqueza observada e esperada para os campos do Marajó}

As coletas realizadas pelo presente estudo entre 2003 e 2005 totalizaram 42 unidades de amostras nas áreas de campos, chamados polígonos de savana da Ilha do Marajó. Nestes três locais foram coligidas 146 espécies, sendo que destas, sendo que destas, 89 espécies (60\%) e oito famílias da classe Actinopterygii foram registradas como novas ocorrências para os campos da Ilha do Marajó, a saber: Achiridae, Cetopsidae, Ctenoluciidae, Paralichthyidae, Poecilidae, Polycentridae, Rivulidae e Trichomycteridae. Com o incremento

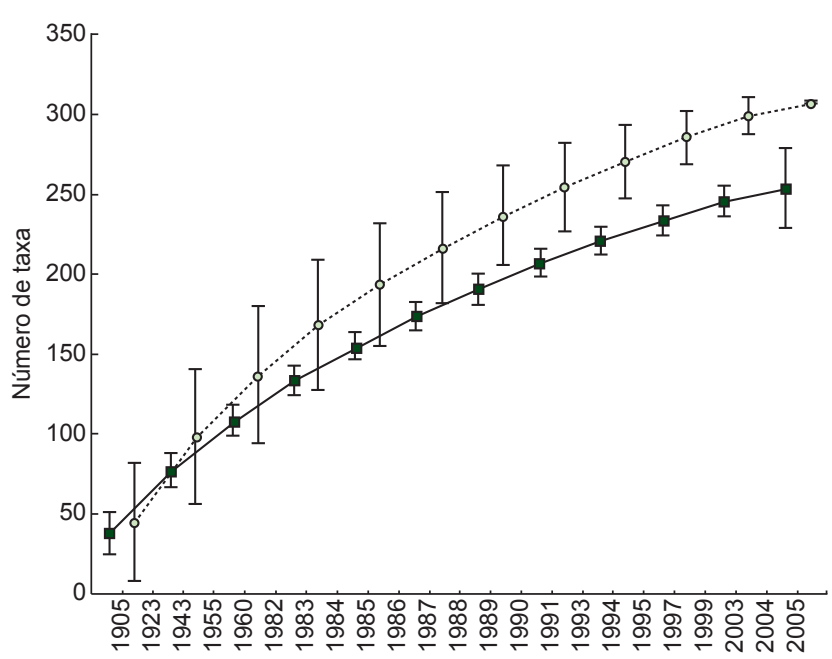

Ano

$$
\begin{aligned}
& \rightarrow \text { - Rarefação (254 spc.) } \\
& \text {--o- Bootstrap (308 spc.) }
\end{aligned}
$$

Figura 2. Curva de acumulação de espécies para os registros dos campos alagados, por técnica de rarefação de amostras, e o número de espécies (riqueza) estimada por técnica de Bootstrap entre os anos de 1905 a 2005 na Ilha do Marajó, Pará, Brasil.

Figure 2. Species accumulation curve to the records of the savannas by technique of rarefaction, and the number of species (richness) estimated by Bootstrap, between the years 1905 and 2005 on the Marajó Island, Pará, Brazil. das coletas nos municípios de Ponta de Pedras, Chaves e Muaná o total de espécies para os campos da ilha é de 254 espécies (Figura 2 e Tabela 2).

A curva de acumulação de espécies, obtida pela técnica de rarefação de amostras, e a estimativa do número de espécies (riqueza estimada), obtido pela técnica de Bootstrap, considerando os registros entre os anos de 1923 a 2005 da ilha do Marajó, indicam que até o ano de 1984, aparentemente o esforço de coleta para os campos do Marajó se apresentaram próximo ao esperado (Figura 2). Uma vez que a curva observada está dentro do intervalo do desvio do esperado de espécie, esta situação foi revertida com um maior esforço de coletas realizadas pela pesquisadora do Museu Paraense Emílio Goeldi, I. Assunção, entre os anos 1985 a 1994, apresentando novas ocorrências para os campos do Marajó, afastando as estimativas do observado. Nos anos subseqüentes esse fato repetir-se-ia, sendo que nos dias atuais obtemos uma estimativa de aproximadamente 308 espécies para as áreas de campos do Marajó, o que representa quase 50 novas ocorrências de espécies para a ilha de Marajó que ainda podem vir a ser catalogadas.

\section{Composição de espécies dos campos do Marajó}

As ordens Characiformes (29\%), Siluriformes (25\%), Perciformes (exclusivos de água doce) (13\%) e Gymnotiformes (13\%) dominam a ictiofauna da região com $86 \%$ das espécies encontradas e depositadas na coleção ictiológica dos museus (Figura 3). Esta composição é semelhante à composição geral da ictiofauna da bacia Amazônica (Lowe-McConnell 1999), e demonstra a natureza fluvial desta fauna apesar da proximidade ao oceano. No período de águas altas, as descargas dos rios Amazonas e Tocantins rodeiam totalmente a Ilha do Marajó, tornando-a uma ilha em águas continentais. No entanto, no período de seca, águas salobras margeiam a costa leste do Marajó, penetrando mais de 100 km na baía do Marajó, mas não chegam a penetrar no interior da ilha (Egler \& Schwassmann 1962, Schwassmann et al. 1989, Barthem \& Schwassmann 1994).

Dos sete municípios com informações a cerca da ictiofauna dos campos do Marajó, Cachoeira do Ararí é o que apresenta a maior riqueza (114 espécies) e com mais de 50\% das espécies exclusivas (Tabela 3). Outros municípios que apresentaram exclusividade maior que $50 \%$ foram Chaves, no extremo norte da ilha, e Salvaterra, no noroeste (Tabela 3).

Atualmente são registrados 1.122 lotes para a ictiofauna dos campos alagados do Marajó, dentre esses registros, as espécies com maior representatividade foram Eigenmannia sp. 1 com 47 lotes, seguido de Trachelyopterus sp. (42 lotes), Pygocentrus nattereri (34 lotes), Hoplias malabaricus (33 lotes), e Sternopygus macrurus (25 lotes). Apenas quatro espécies ocorreram em todas as localidades são elas: Astyanax bimaculatus, Hoplias malabaricus. Hyphessobrycon sp. e Plagioscion squamosissimus. Até o presente momento, nenhuma espécie de peixe é considerada endêmica para os campos do Marajó, porém vale ressaltar que muitas espécies apresentam deficiência na sua identificação a nível específico, devido a carência de estudos sistemáticos e taxonômicos em diversos grupos.

As famílias Characidae (Characiformes) e Cichlidae (Perciformes) foram as mais representativas com $16 \%$ e $10 \%$ do numero total de espécies, respectivamente. Também se destacaram outras famílias, como Loricariidae (Siluriformes) com 7\%, Apteronotidae (Gymnotiformes) e Auchenipteridae (Siluriformes) com aproximadamente 5\% das espécies cada.

\section{Status de conservação da ictiofauna dos campos alagados do Marajó}

Em decorrência da pesca excessiva e das modificações de hábitat, provenientes da intensa atividade pecuarista e de construções de hi- 
Tabela 2. Compilação dos dados secundários (anos de 1923 a 1999) e primários (anos de 2003 a 2005) das ordens, famílias e nomes científicos das espécies de peixes dos campos alagados da Ilha do Marajó, Pará, Brasil.

Table 2. Secondary data (1923 to 1999) and primary data (2003 to 2005) of orders, families, and fish species in flooded áreas of Marajó Island, Pará, Brazil.

Espécies

\section{Beloniformes}

\section{BELONIDAE}

Potamorrhaphis guianensis (Jardine, 1843)

Potamorrhaphis sp.

\section{Characiformes}

ACESTRORHYNCHIDAE

Acestrorhynchus altus Menezes, 1969

Acestrorhynchus cf. altus Menezes, 1969

Acestrorhynchus falcatus (Bloch 1794)

Acestrorhynchus falcirostris (Cuvier, 1819)

Acestrorhynchus microlepis (Schomburgk, 1841)

Acestrorhynchus sp.

ANOSTOMIDAE

Leporinus affinis Günther, 1864

Leporinus fasciatus (Bloch, 1794)

Leporinus friderici (Bloch, 1794)

Leporinus sp.

Rhytiodus microlepis Kner, 1858

Schizodon fasciatus Spix \& Agassiz, 1829

Schizodon sp.

Schizodon vittatus (Valenciennes, 1850)

CHARACIDAE

Astyanax bimaculatus (Linnaeus, 1758)

Bryconops alburnoides Kner, 1858

Bryconops caudomaculatus (Günther, 1864)

Bryconops giacopinii (Fernández-Yepez, 1950)

Bryconops sp.

Charax pauciradiatus (Günther, 1864)

Cheirodon sp.

Hemigrammus bellottii (Steindachner, 1882)

Hemigrammus levis Durbin, 1908

Hemigrammus ocellifer (Steindachner, 1882)

Hemigrammus rhodostomus Ahl, 1924

Hemigrammus sp.

Hemigrammus unilineatus (Gill, 1858)

Hyphessobrycon heterorhabdus (Ulrey, 1894)

Hyphessobrycon sp.

Metynnis luna Cope, 1878

Metynnis sp.

Moenkhausia collettii (Steindachner, 1882)

Moenkhausia lepidura (Kner, 1858)

Moenkhausia pyrophthalma Costa, 1994 $\mathrm{x} \quad \mathrm{x}$

$\mathrm{x}$

$\mathrm{x}$

$\mathrm{x}$

$\mathrm{X}$

$\mathrm{X}$

$\mathrm{X}$

$\mathrm{X}$
Marajó

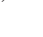

Espécies

Moenkhausia sp.

Paragoniates alburnus Steindachner, 1876

Phenacogaster sp.

Poptella sp.

Pristella maxillaris (Ulrey, 1894)

Pristobrycon aureus (Spix \& Agassiz, 1829)

Pristobrycon calmoni (Steindachner, 1908)

Pygocentrus nattereri Kner, 1858

Roeboides myersii Gill, 1870

Roeboides sp.

Serrasalmus elongatus Kner, 1858

Serrasalmus rhombeus (Linnaeus, 1766)

Serrasalmus sp.

Triportheus aff. angulatus (Spix \& Agassiz, 1829)

Triportheus albus Cope, 1872

Triportheus angulatus (Spix \& Agassiz, 1829)

Triportheus elongatus (Günther, 1864)

Triportheus sp.

CRENUCHIDAE

Crenuchus spilurus Günther, 1863

Microcharacidium sp.

CTENOLUCIIDAE

Boulengerella lucius (Cuvier, 1816) X

CURIMATIDAE

Curimata inornata Vari, 1989

Curimata sp.1

Curimata sp.2

Curimatopsis sp. ERYTHRINIDAE

Erythrinus erythrinus (Bloch \& Schneider, 1801)

Hoplerythrinus unitaeniatus (Spix \& Agassiz, 1829)

Hoplias malabaricus (Bloch, 1794)

Hoplias sp. GASTEROPELECIDAE

Carnegiella sp.

Carnegiella strigata (Günther, 1864)

Gasteropelecus sternicla (Linnaeus, 1758)

Thoracocharax stellatus (Kner, 1858)

HEMIODONTIDAE

Hemiodus unimaculatus (Bloch, 1794)

LEBIASINIDAE

Copella arnoldi (Regan, 1912)

Copella nattereri (Steindachner, 1876)

Nannostomus eques Steindachner, 1876

Nannostomus nittidus Wetziman, 1978

Pyrrhulina filamentosa Valenciennes, 1847
$\mathrm{X}$

.

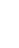

$\mathrm{x}$


Tabela 2. Continuação...

Espécies

\section{Clupeiformes \\ ENGRAULIDIDAE}

Anchovia sp.

Anchovia surinamensis (Bleeker, 1866)

Lycengraulis batesii (Günther, 1868)

Lycengraulis sp.

Pterengraulis atherinoides (Linnaeus, 1766)

PRISTIGASTERIDAE

Pellona castelnaeana (Valenciennes, 1847)

Pellona flavipinnis (Valenciennes, 1836)

Pellona sp.

\section{Cyprinodontiformes}

ANABLEPIDAE

Anableps microlepis Muller \& Troschel, 1844

POECILIIDAE

Pamphorichthys sp.

Poecilia sp.1

Poecilia sp.2

Poecilia sp. 3

Poecilia sp.4

Poecilia sp.5

RIVULIDAE

Rivulus sp.

\section{Gymnotiformes}

Apteronotidae

Adontosternarchus sp.

Apteronotus albifrons (Linnaeus, 1766)

Apteronotus sp.

Sternarchella cf. terminalis

(Eigenmann \& Allen, 1942)

Sternarchella sp.

Sternarchogiton nattereri (Steindachner, 1868)

Sternarchogiton porcinum Eigenmann \& Allen, 1942

Sternarchogiton sp.

Sternarchorhamphus muelleri (Steindachner, 1881)

Sternarchorhamphus sp.

GYMNOTIDAE

Electrophorus electricus (Linnaeus, 1766)

Gymnotus carapo Linnaeus, 1758

Gymnotus sp.

\section{HYPOPOMIDAE}

Brachyhypopomus brevirostris (Steindachner, 1868)

Brachyhypopomus pinnicaudatus (Hopkins, 1991)

Brachyhypopomus sp.

Hypopomus sp.

Hypopygus lepturus Hoedeman, 1962

$\mathrm{X}$
Tabela 2. Continuação...

\begin{tabular}{|c|c|c|}
\hline Espécies & 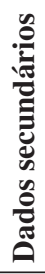 & 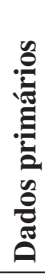 \\
\hline Hypopygus sp. & & $\mathrm{x}$ \\
\hline Microsternarchus bilineatus Fernández-Yépez, 1968 & & $\mathrm{x}$ \\
\hline Steatogenys elegans (Steindachner, 1880) & $\mathrm{x}$ & $\mathrm{x}$ \\
\hline Steatogenys sp. & & $\mathrm{x}$ \\
\hline RHAMPHICHTHYIDAE & & \\
\hline Rhamphichthys marmoratus Castelnau, 1855 & & $\mathrm{x}$ \\
\hline Rhamphichthys rostratus (Linnaeus, 1766) & $\mathrm{x}$ & \\
\hline Rhamphichthys sp. & $\mathrm{x}$ & \\
\hline STERNOPYGIDAE & & \\
\hline Distocyclus conirostris (Eigenmann \& Allen, 1942) & $\mathrm{x}$ & \\
\hline Eigenmannia humboldtii (Steindachner, 1878) & $\mathrm{x}$ & \\
\hline Eigenmannia sp.1 & $\mathrm{x}$ & \\
\hline Eigenmannia sp.2 & & $\mathrm{x}$ \\
\hline Rhabdolichops sp. & $\mathrm{x}$ & \\
\hline Rhabdolichops troscheli (Kaup, 1856) & $\mathrm{x}$ & \\
\hline Sternopygus macrurus (Bloch \& Schneider, 1801) & $\mathrm{x}$ & $\mathrm{x}$ \\
\hline Sternopygus sp. & & $\mathrm{x}$ \\
\hline
\end{tabular}

\section{Lepidosireniformes}

LEPIDOSIRENIDAE

Lepidosiren paradoxa Fitzinger, 1837

$\mathrm{X}$

Osteoglossiformes

ARAPAIMIDAE

Arapaima gigas (Schinz, 1822)

Osteoglossidae

Osteoglossum bicirrhosum (Cuvier, 1829)

$\mathrm{X}$

Perciformes

CENTROPOMIDAE

Centropomus sp.

CICHLIDAE

Acaronia nassa (Heckel, 1840)

Aequidens pallidus (Heckel, 1840)

Aequidens sp.

Apistogramma luelingi Kullander, 1976

Apistogramma sp.

Astronotus ocellatus (Agassiz, 1831)

Chaetobranchopsis orbicularis (Steindachner, 1875)

Chaetobranchopsis sp.

Chaetobranchus sp.

Cichla melaniae Kullander \& Ferreira, 2006 x

Cichla monoculus Spix \& Agassiz, 1831

Cichla nigromaculata Jardine, 1843

Cichla ocellaris Bloch \& Schneider, 1801

$\mathrm{X}$

X $\quad \mathrm{X}$

$\mathrm{X} \quad \mathrm{X}$

$\mathrm{X}$

$\mathrm{X}$

$\mathrm{X} \quad \mathrm{X}$

$\mathrm{X}$

$\mathrm{X}$

$\mathrm{X}$

$\mathrm{X}$

$\mathrm{X} \quad \mathrm{X}$

$\mathrm{X}$

$\mathrm{X}$

Cichla sp.1

Cichla sp. 2

Cichla temensis Humboldt, 1821

$\mathrm{X}$

$\mathrm{X}$

$\mathrm{X} \quad \mathrm{X}$

$\mathrm{X}$ 
Tabela 2. Continuação...

\begin{tabular}{|c|c|c|}
\hline Espécies & 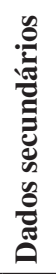 & 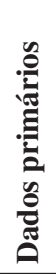 \\
\hline Crenicichla cincta Regan, 1905 & $\mathrm{x}$ & $\mathrm{x}$ \\
\hline Crenicichla macrophthalma Heckel, 1840 & & $\mathrm{x}$ \\
\hline Crenicichla notophthalmus Regan, 1913 & & $\mathrm{x}$ \\
\hline Crenicichla regani Ploeg, 1991 & $\mathrm{x}$ & $\mathrm{x}$ \\
\hline Crenicichla reticulata Heckel, 1840 & & $\mathrm{x}$ \\
\hline Crenicichla sp.1 & & $\mathrm{x}$ \\
\hline Crenicichla sp.2 & & $\mathrm{x}$ \\
\hline Crenicichla sp.3 & & $\mathrm{x}$ \\
\hline Crenicichla strigata Günther, 1862 & & $\mathrm{x}$ \\
\hline Geophagus camopiensis Pellegrin, 1903 & $\mathrm{x}$ & \\
\hline Geophagus sp. & $\mathrm{x}$ & $\mathrm{x}$ \\
\hline Geophagus surinamensis (Bloch, 1791) & & $\mathrm{x}$ \\
\hline Satanoperca jurupari (Heckel, 1840) & & $\mathrm{x}$ \\
\hline GOBIIDAE & & \\
\hline Awaous flavus (Valenciennes, 1837) & $\mathrm{x}$ & \\
\hline Bathygobius sp. & $\mathrm{x}$ & \\
\hline Eleotris sp. & & $\mathrm{x}$ \\
\hline Evorthodus sp. & & $\mathrm{x}$ \\
\hline Gobioides sp. & & $\mathrm{x}$ \\
\hline Gobionellus sp. & & $\mathrm{x}$ \\
\hline MUGILIDAE & & \\
\hline Mugil curema Valenciennes, 1836 & $\mathrm{x}$ & $\mathrm{x}$ \\
\hline Polycentridae & & \\
\hline Monocirrhus polyacanthus Heckel, 1840 & & $\mathrm{x}$ \\
\hline Polycentrus schomburgkii Müller \& Troschel, 1848 & & $\mathrm{x}$ \\
\hline SCIAENIDAE & & \\
\hline Cynoscion microlepidotus (Cuvier, 1830) & $\mathrm{x}$ & \\
\hline Cynoscion sp. & $\mathrm{x}$ & \\
\hline Macrodon ancylodon (Bloch \& Schneider, 1801) & $\mathrm{x}$ & \\
\hline Pachypops fourcroi (Lacepède, 1802) & & $\mathrm{x}$ \\
\hline Pachypops sp. & & $\mathrm{x}$ \\
\hline Pachypops trifilis (Müller \& Troschel, 1849) & & $\mathrm{x}$ \\
\hline Plagioscion auratus (Castelnau, 1855) & $\mathrm{x}$ & \\
\hline Plagioscion sp. & $\mathrm{x}$ & \\
\hline Plagioscion squamosissimus (Heckel, 1840) & $\mathrm{x}$ & $\mathrm{x}$ \\
\hline Plagioscion surinamensis (Bleeker, 1973) & $\mathrm{x}$ & \\
\hline Pleuronectiformes & & \\
\hline Achiridae & & \\
\hline Achirus achirus (Linnaeus, 1758) & & $\mathrm{x}$ \\
\hline Achirus sp. & & $\mathrm{x}$ \\
\hline PARALICHTHYIDAE & & \\
\hline Paralichthys brasiliensis (Ranzani, 1842) & & $\mathrm{x}$ \\
\hline Paralichthys sp. & & $\mathrm{x}$ \\
\hline Rajiformes & & \\
\hline POTAMOTRYGONIDAE & & \\
\hline Potamotrygon motoro (Müller \& Henle, 1841) & $\mathrm{x}$ & \\
\hline
\end{tabular}

Tabela 2. Continuação...

\begin{tabular}{|c|c|c|}
\hline Espécies & 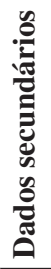 & 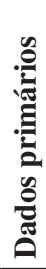 \\
\hline Potamotrygon orbignyi (Castelnau, 1855) & $\mathrm{x}$ & $\mathrm{x}$ \\
\hline Potamotrygon scobina Garman, 1913 & & $\mathrm{x}$ \\
\hline Potamotrygon sp. & $\mathrm{x}$ & \\
\hline \multicolumn{3}{|l|}{ Siluriformes } \\
\hline \multicolumn{3}{|l|}{ ASPREDINIDAE } \\
\hline Aspredinichthys filamentosus (Valenciennes, 1840) & $\mathrm{x}$ & \\
\hline Aspredo aspredo (Linnaeus, 1758) & $\mathrm{x}$ & \\
\hline Bunocephalus aleuropsis Cope, 1870 & & $\mathrm{x}$ \\
\hline \multicolumn{3}{|l|}{ AUCHENIPTERIDAE } \\
\hline Ageneiosus inermis (Linnaeus, 1766) & $\mathrm{x}$ & \\
\hline Ageneiosus sp.1 & $\mathrm{x}$ & \\
\hline Ageneiosus sp.2 & $\mathrm{x}$ & $\mathrm{x}$ \\
\hline Ageneiosus ucayalensis Castelnau, 1855 & $\mathrm{x}$ & $\mathrm{x}$ \\
\hline Asterophysus sp. & $\mathrm{x}$ & \\
\hline Auchenipterichthys longimanus (Günther, 1864) & & $\mathrm{x}$ \\
\hline Auchenipterus nuchalis (Spix \& Agassiz, 1829) & $\mathrm{x}$ & \\
\hline Centromochlus heckelii (De Filippi, 1853) & & $\mathrm{x}$ \\
\hline Pseudauchenipterus nodosus (Bloch, 1794) & $\mathrm{x}$ & \\
\hline Tatia intermedia (Steindachner, 1877) & $\mathrm{x}$ & \\
\hline Trachelyopterus galeatus (Linnaeus, 1766) & $\mathrm{x}$ & $\mathrm{x}$ \\
\hline Trachelyopterus sp. & $\mathrm{x}$ & \\
\hline \multicolumn{3}{|l|}{ CALLICHTHYIDAE } \\
\hline Callichthys callichthys (Linnaeus, 1758) & $\mathrm{x}$ & \\
\hline Hoplosternum littorale (Hancock, 1828) & $\mathrm{x}$ & $\mathrm{x}$ \\
\hline Megalechis personata (Ranzini, 1841) & $\mathrm{x}$ & \\
\hline Megalechis thoracata (Valenciennes, 1840) & $\mathrm{x}$ & $\mathrm{x}$ \\
\hline \multicolumn{3}{|l|}{ CETOPSIDAE } \\
\hline Cetopsis sp. & & $\mathrm{x}$ \\
\hline Hemicetopsis sp. & & $\mathrm{x}$ \\
\hline \multicolumn{3}{|l|}{ DORADIDAE } \\
\hline Acanthodoras sp. & $\mathrm{x}$ & \\
\hline Anadoras sp. & $\mathrm{x}$ & \\
\hline Doras eigenmanni (Boulenger, 1895) & $\mathrm{x}$ & \\
\hline Doras sp. & $\mathrm{x}$ & \\
\hline Hassar sp. & $\mathrm{x}$ & \\
\hline Lithodoras dorsalis (Valenciennes, 1840) & $\mathrm{x}$ & \\
\hline \multicolumn{3}{|l|}{ HEPTAPTERIDAE } \\
\hline Gladioglanis machadoi Ferraris \& Mago-Leccia, 1989 & & $\mathrm{x}$ \\
\hline Pimelodella altipinnis (Steindachner, 1864) & $\mathrm{x}$ & $\mathrm{x}$ \\
\hline Pimelodella cristata (Müller \& Troschel, 1848) & $\mathrm{x}$ & $\mathrm{x}$ \\
\hline Pimelodella gracilis (Valenciennes, 1835) & & $\mathrm{x}$ \\
\hline Pimelodella sp. & $\mathrm{x}$ & \\
\hline Rhamdia quelen (Quoy \& Gaimard, 1824) & $\mathrm{x}$ & \\
\hline Rhamdia sp. & & $\mathrm{x}$ \\
\hline Insertae sedis & & \\
\hline Phreatobius cisternarum Goeldi, 1905 & $\mathrm{x}$ & \\
\hline
\end{tabular}


Tabela 2. Continuação...

Espécies

Loricariidae

Ancistrus sp. 1

Ancistrus sp. 2

Chaetostoma sp.

Farlowella aff. knerii (Steindachner, 1882)

Farlowella amazona (Gunther, 1864)

Farlowella sp.

Hemiancistrus sp.

Hemiodontichthys acipenserinus (Kner, 1853)

Hemiodontichthys sp.

Hypoptopoma sp.

Hypostomus sp.

Lasiancistrus sp.

Limatulichthys sp.

Liposarcus pardalis (Castelnau, 1855)

Loricaria catphracta Linnaeus, 1758

Panaque sp.

Pseudoloricaria sp.

Pterygoplichthys sp.

Reganella sp.

PIMELODIDAE

Brachyplatystoma vaillantii (Valenciennes, 1840)

Hypophthalmus marginatus Valenciennes, 1840

Pimelodus blochii Valenciennes, 1840
Tabela 2. Continuação...

\begin{tabular}{|c|c|c|}
\hline Espécies & 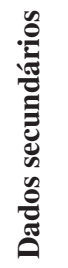 & 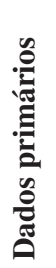 \\
\hline Pimelodus ornatus Kner, 1858 & $\mathrm{x}$ & $\mathrm{x}$ \\
\hline Pimelodus sp.1 & $\mathrm{x}$ & $\mathrm{x}$ \\
\hline Pimelodus sp.2 & & $\mathrm{x}$ \\
\hline Pinirampus pirinampu (Spix \& Agassiz, 1829) & & $\mathrm{x}$ \\
\hline Zungaro zungaro (Humboldt, 1821) & $\mathrm{x}$ & $\mathrm{x}$ \\
\hline PSEUDOPIMELODIDAE & & \\
\hline Batrochoglanis raninus (Valenciennes, 1840) & $\mathrm{x}$ & $\mathrm{x}$ \\
\hline TRICHOMYCTERIDAE & & \\
\hline Henonemus taxistigmus (Fowler, 1814) & & $\mathrm{x}$ \\
\hline Synbranchiformes & & \\
\hline SYNBRANCHIDAE & & \\
\hline $\begin{array}{l}\text { Synbranchus lampreia } \\
\text { Favorito, Zanata \& Assumpção, } 2005\end{array}$ & $\mathrm{x}$ & \\
\hline Synbranchus marmoratus Bloch, 1795 & $\mathrm{x}$ & $\mathrm{x}$ \\
\hline Synbranchus sp. & $\mathrm{x}$ & \\
\hline Tetraodontiformes & & \\
\hline TETRAODONTIDAE & & \\
\hline Colomesus asellus (Müller \& Troschel, 1849) & & $\mathrm{x}$ \\
\hline Colomesus psittacus (Bloch \& Schneider, 1801) & $\mathrm{x}$ & $\mathrm{x}$ \\
\hline Total de espécies & 165 & 146 \\
\hline No espécies exclusivas & 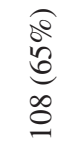 & $\begin{array}{l}\widehat{\rho} \\
0 \\
\stackrel{0}{0} \\
\infty\end{array}$ \\
\hline
\end{tabular}

manejo adequado para várias regiões da Amazônia, com a ocorrência de sobrepesca para alguns estoques pesqueiros, como "tambaqui" Colossoma macropomum (Cuvier, 1818) (Isaac \& Ruffino 1996) e "piramutaba" Brachyplatystoma vaillantii (Valenciennes, 1840) (Barthem \& Petrere 1995). A principal pesca que se dá no Marajó é a do "tamoatá" Hoplosternun spp. (Siluriformes: Callichthyidae), cujo recurso tem sido manejado com a implantação do período de defeso (Albuquerque 2006). Mas há pouca informação sobre os resultados deste manejo.

Das 254 espécies registradas até o presente momento nas savanas do Arquipélago do Marajó, 44 espécies (17\% do total) são alvos da pesca comercial para fins de alimentação e 46 espécies (18\%) são capturadas na pesca para ornamental. Como citado por Barthem e Fabré (2003) os campos da Ilha de Marajó e da costa do Amapá são as áreas mais importantes de pesca da região, as quais são alagadas por chuvas locais.

Apenas três espécies da ictiofauna dos campos estão em situação de risco de ameaça ou não se tem dados suficientes para avaliá-las, são eles: o "pirarucu" (Arapaima gigas) (Schinz, 1822) e as "raias ou arraias" Potamotrygon motoro e P. scobina (German, 1913).

Essas espécies estão classificadas na categoria de deficiência de dados da IUCN (International Union for Conservation of Nature), pois não possuem dados suficientes para uma avaliação adequada sobre possível risco de extinção, não tendo como definir sua distribuição 
Tabela 3. Número de espécies (riqueza) e de espécies "exclusivas" para os sete municípios que há dados sobre a ictiofauna dos campos do Arquipélago do Marajó, Pará, Brasil.

Table 3. Richness and 'exclusive' species of ichthyofauna from seven municipalities of Marajó Island, Pará, Brazil.

\begin{tabular}{|c|c|c|c|c|c|c|c|}
\hline & & & & Municípios & & & \\
\hline Indicador & $\begin{array}{l}\text { Cachoeira } \\
\text { do Arari }\end{array}$ & Chaves & Muaná & $\begin{array}{l}\text { Ponta de } \\
\text { Pedras }\end{array}$ & Salvaterra & $\begin{array}{c}\text { Santa Cruz } \\
\text { do Arari }\end{array}$ & Soure \\
\hline Riqueza & 144 & 47 & 62 & 76 & 23 & 11 & 4 \\
\hline Exclusividades & $83(58 \%)$ & $24(51 \%)$ & $23(37 \%)$ & $34(45 \%)$ & $12(52 \%)$ & $2(18 \%)$ & $1(25 \%)$ \\
\hline
\end{tabular}

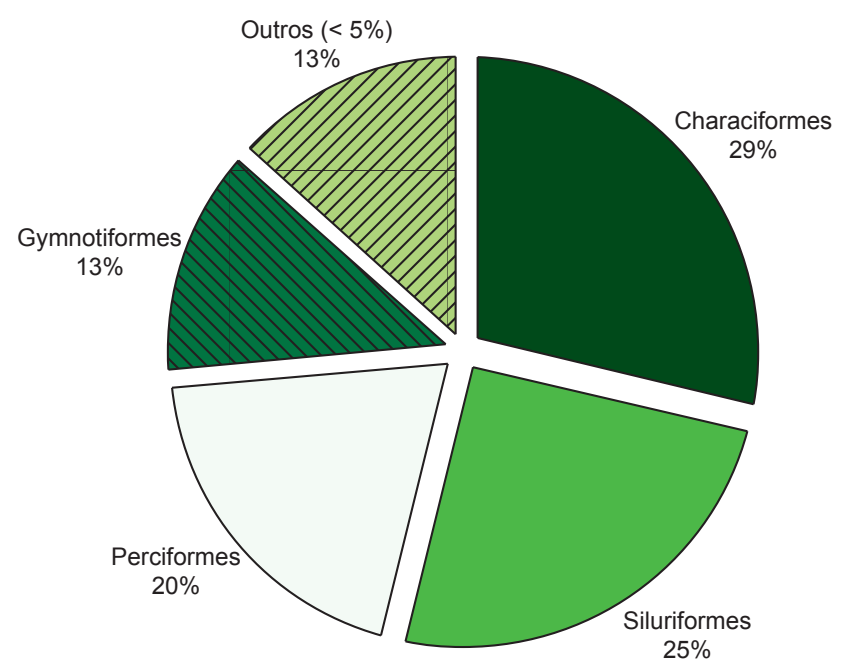

Figura 3. Representatividade das espécies de peixes dentro das ordens para os campos alagados do Arquipélago do Marajó, Pará, Brasil. Outros representam ordens com menos de 5\% de representatividade, são elas: Clupeiformes, Cyprinodontiformes, Pleuronectiformes, Rajiformes, Synbranchiformes, Beloniformes, Osteoglossiformes, Tetraodontiformes e Lepidosireniformes.

Figure 3. Representativeness of fish species within the orders for the areas of savannas of the Marajó Island, Pará, Brazil. Other orders represent less than 5\%, so they are: Clupeiformes, Cyprinodontiformes, Pleuronectiformes, Rajiformes, Synbranchiformes, Beloniformes, Osteoglossiformes, Tetraodontiformes and Lepidosireniformes.

e abundância de suas populações. Desta forma, seriam necessário maiores informações sobre essas espécies para se obter uma melhor classificação quanto ao seu risco de extinção.

Apesar das lacunas existentes no inventário da fauna de peixes dos campos do Marajó e da precariedade das identificações em nível de espécie ocasionada pela falta de estudos de revisão sistemática da maioria dos grupos, os dados atualmente disponíveis possibilitam assinalar, que áreas de campo do Marajó, são áreas prioritárias para conservação e de inventários biológicos com base em critérios bem definidos.

Assim, fica claro que há ainda um longo caminho a ser percorrido no que diz respeito à documentação da fauna dos ambientes aquáticos, através de coletas, identificação e catalogação de espécies em áreas pouco exploradas, principalmente nas cabeceiras de rios e igarapés, caracterizadas por elevado grau de endemismo. Sem esta documentação, qualquer avaliação de biodiversidade, delimitação de áreas de endemismo e distribuição geográfica para execução de medidas conservacionistas fica prejudicada. É necessário, portanto, não apenas criar condições para proteger a fauna, mas para torná-la melhor conhecida através da criação de mecanismos de apoio a projetos de inventariamento e estudos de taxonomia e sistemática.

\section{Agradecimentos}

Os autores são gratos ao Projeto de Conservação e Utilização Sustentável da Diversidade Biológica (PROBIO) do Ministério do Meio Ambiente, subsidiado pelo Global Environment Facility (GEF), o qual financiou o projeto "Avaliação Ecológica e Seleção de Áreas Prioritárias à Conservação de Savanas Amazônicas, Arquipélago do Marajó, Estado do Pará” (PROBIO Marajó).

\section{Referências Bibliográficas}

ALBUQUERQUE, A. 2006. A pesca do tamoatá Hoplosternum littorale (Hancocck, 1828) Siluriformes: Callichthyidae), na ilha de Marajó. Dissertação de Mestrado, Universidade Federal do Pará/Museu Paraense Emílio Goeldi, 59 p.

BARTHEM, R.B. \& SCHWASSMANN, H.O. 1994. The Amazon river influence over the seasonal displacement of the salty wedges in Tocantins estuary, Brazil, 1983-1985. Bol. Mus. Para. Emílio Goeldi. 10(1):119-130.

BARTHEM, R.B. \& FABRÉ N.N. 2003. Biologia e diversidade dos recursos pesqueiros da Amazônia. In A pesca e os recursos pesqueiros na Amazônia brasileira (M.L. Rufino, ed.). Pro-Várzea, Manaus, p. 11-55.

BARTHEM, R.B. \& PETRERE Jr., M. 1995. Fisheries and populations dynamics of the freshwater catfish Brachyplatystoma vaillantii in the Amazon estuary. In Condition of the World's Aquatic Habitat (N.B Armantrout, ed.). Proceedings of the World Fisheries Congress: theme 1. Oxford \& IBH Publishing Co. Pvt. Ltd., New Delhi, p. 329-350.

BOULENGER, G.A. 1897. On a collection of fishes from the island of Marajó, Brazil. Ann. Mag. Nat. Hist. 20(117):294-299.

BRASIL, M.C. 1999. Marajó: em busca da sobrevivência. Textos IESAM, Belém, Pará, p. 80. (n. 7).

BUCKUP, P.A. 1993. Review of the characidiin fishes (Teleostei: Characiformes) with descriptions of four new genera and ten new species. Ichthyol. Explor. Freshw. Cornol. 4(2):97-154.

BUCKUP, P.A., MENEZES, N.A. \& GHAZZI, M.S. 2007. Catálogo das espécies de peixes de água doce do Brasil. Museu Nacional, Rio de Janeiro, 195 p. (série livros, n. 23).

BURGESS, W.E. 1989. An atlas of freshwater and marine catfishes: a preliminary survey of the Siluriformes. T.F.H., Nepture City, 784 p.

COLWELL, R.K. 1997. User's guide to Estimates 5 statistical: estimation of species richness and shared species from samples. Version 5.0.1. University of Connecticut, Storrs, $22 \mathrm{p}$.

COLWELL, R.K. \& CODDINGTON, J.A. 1994. Estimating terrestrial biodiversity through extrapolation. Phil. Trans. R. Soc. Lond. B. 345:101-118

CRUZ, M.E.M. 1987. Marajó, essa imensidão de ilha. São Paulo, 111 p.

EGLER, W.A. \& SCHWASSMANN, H.O. 1962. Limnological studies in the Amazon Estuary. Bol. Mus. Para. Emílio Goeldi, nova sér., 1:2-25.

ELLIS, M.M. 1913. The gymnotid eels of tropical America. Mem. Carneg. Mus. 6(3):109-195.

FISHBASE. www.fishbase.org (último acesso em 01/11/2007). 
GÉRY, J. 1977. Characoids of the world. T.F.H. Publications, Inc. Ltd., Neptune City, 672 p.

GOULDinG, M., BARTHEM, R. \& FERREIRA, E.J.G. 2003. The Smithsonian Atlas of the Amazon. Smithsonian Institution, Washington, $253 \mathrm{p}$

Instituto Brasileiro de Geografia e Estatística - IBGE. 1991. Geografia do Brasil: região norte. IBGE/Diretoria de Geociências, Rio de Janeiro, p. 1-307. (v. 3).

ISAAC, V.J. \& RUFFINO, M.L. 1996. Population dynamics of tambaqui Colossoma macropomum Cuvier 1818, in the Lower Amazon, Brazil. Fish. Manag. Ecol. 3:315-333.

ISBRÜCKER, I.J.H. 1981. Revision of Loricaria Linnaeus, 1758 (Pisces, Siluriformes, Loricariidae). Beaufortia. 31(3):51-96.

LOWE-McCONNELL, R.H. 1999. Estudos ecológicos de comunidades de peixes tropicais. EDUSP, São Paulo, 536 p.

MAGO-LECCIA, F. 1994. Electric fishes of the continental waters of America. Ed. Clemente, Venezuela, 206 p.

MIRANDA-NETO, M.J. 1968. A Foz do Rio-Mar: subsídios para o desenvolvimento de Marajó. Distribuidora Record, Rio de Janeiro, $197 \mathrm{p}$.

Museu Paraense Emílio Goeldi - MPEG. 2005. Programa de Biodiversidade da Amazônia. http://www.museu-goeldi.br/biodiversidade/index.asp (último acesso em 01/01/2006).
NASCIMENTO, F.P., Ávila-Pires, T.C.S., Santos, I.N.F. \& Lima, A.C.M. 1991. Répteis de Marajó e Mexiana, Pará, Brasil. Revisão bibliográfica e novos registros. Bol. Mus. Para. Emílio Goeldi, série Zoologia, 7(1):25-41.

REIS, R.E. 1997. Revision of the Neotropical catfish genus Hoplosternum (Osteriophysi: Siluriformes: Callichthyidae), with the description of two new genera and three new species. Ichthyol. Explor. Freshw. 7(4):299326.

REIS, R.E., KULLANDER, S.O. \& FERRARIS Jr., C.J. 2003. Check list of freshwater fishes of South and Central America. EDIPUCRS, Porto Alegre, $742 \mathrm{p}$.

Schwassmann, H.O., Barthem, R.B. \& Carvalho, M.L. 1989. A note on the seasonally shifting zone of high primary production in the bay of Marajó, Pará, Brazil, 1983-1984. Acta Bot. Bras. 2(1):165-174.

Secretaria Executiva de Estado de Planejamento, Orçamento e Finanças SEPOF. 2007. Estatística Municipal. Diretoria de Estudos, Pesquisas e Informações Sócio-Econômicas, Chaves, Pará, 43 p.

SIBIP/NEODAT III. www.mnrj.ufrj.br/search1c.htm (último acesso em 01/11/2007).

SIOLI, H. 1966. General features of the delta of the Amazon. In Humid tropics research: scientific problems of the humid tropical zone deltas and their implications. Proceedings Dacca Symposium. UNESCO, New York, p. 381-390.

Recebido em 18/02/08

Versão Reformulada recebida em 15/05/09

Publicado em 24/07/09 
Apêndice 1. Material Analisado.

Appendix 1. Analyzed Material.

Beloniformes: BELONIDAE: Potamorrhaphis eigenmanni MPEG 7997; Potamorrhaphis guianensis MPEG 9058; Potamorrhaphis sp. MPEG 2607, MPEG 2817, MPEG 9059, MPEG 9060, MPEG 9061, MPEG 9062, MPEG 9071.

Ch a r a c if or mes: A C E S T ROR H Y N C H ID A E : Acestrorhynchus altus MPEG 2368, MPEG 2716, MPEG 2614, MPEG 2643, MPEG 2906, MPEG 2825, MPEG 7968 , MPEG 2501, MPEG 2467, MPEG 2470, MPEG 2502, MPEG 2592, MPEG 2751; Acestrorhynchus cf. altus MPEG 7982; Acestrorhynchus falcatus MPEG 1708; MPEG 2766, MPEG 2824, MPEG 4825; Acestrorhynchus falcirostris MPEG 7 5 54, MPEG 7969, MPEG 8011; Acestrorhynchus sp. MPEG 2826, MPEG 2603; ANOSTOMIDAE: Leporinus affinis MPEG 7545, MPEG 7971; Leporinus fasciatus MPEG 8003, MPEG 8030; Leporinus friderici MPEG 2367, MPEG 2591, MPEG 2626, MPEG 2758, MPEG 2768, MPEG 2769, MPEG 2844, MPEG 2845, MPEG 2933, MPEG 4855 , MPEG 7984; Leporinus sp. MPEG 4688, MPEG 1643, MPEG 1644, MPEG 1653, MPEG 2530, MPEG 2533, MPEG 2567, MPEG 2531, MPEG 2532, MPEG 2757; Rhytiodus microlepis MPEG 7972; Schizodon fasciatus MPEG 2847, MPEG 2848, MPEG 2934, MPEG 4686, MPEG 1651, MPEG 2566, MPEG 2529; Schizodon sp. MPEG 2055, MPEG 2056, MPEG 2366, MPEG 2534, MPEG 2535 , MPEG 2590, MPEG 5470, MPEG 2625, MPEG 2896; Schizodon vittatus MPEG 2759, MPEG 2767, MPEG 2846, MPEG 4854; CHARACIDAE: Astyanax bimaculatus MPEG 1862, MPEG 1341, MPEG 1885, MPEG 2717, MPEG 2644, MPEG 2635, MPEG 2762 , MPEG 2898, MPEG 2827, MPEG 2935, MPEG 6424, MPEG 6421 , MPEG 7555, MPEG 8018, MPEG 9033, MPEG 9048; Bryconops alburnoides MPEG 4241; Bryconops caudomaculatus MPEG 7584, MPEG 7552, MPEG 7952, MPEG 7995, MPEG 8013; Bryconops giacopinii MPEG 2828, MPEG 7638; Bryconops sp. MPEG 9027, MPEG 8016, MPEG 8025; Charax pauciradiatus MPEG 1874; Cheirodon sp. MPEG 9083; Hemigrammus bellottii MPEG 8024; Hemigrammus levis MPEG 2831; Hemigrammus ocellifer MPEG 7628

MPEG 7629, MPEG 7630, MPEG 7965; Hemigrammus rhodostomus MPEG 2838; Hemigrammus sp. MPEG 2834, MPEG 2452, MPEG 2829, MPEG 2833, MPEG 9044, MPEG 9045, MPEG 9047; Hemigrammus unilineatus MPEG 2832, MPEG 7622; Hyphessobrycon heterorhabdus MPEG 7618, MPEG 7623, MPEG 7624; Hyphessobrycon sp. MPEG 1017, MPEG 2723, MPEG 2636, MPEG 2835, MPEG 7646, MPEG 7647, MPEG 9029, MPEG 9038, MPEG 9051, MPEG 9084, MPEG 7993, MPEG 8022, MPEG 8044; Metynnis luna MPEG 1534, MPEG 2773, MPEG 2856; Metynnis sp. MPEG 2772, MPEG 7556; Moenkhausia collettii MPEG 7639, MPEG 7640; Moenkhausia lepidura MPEG 7636, MPEG 7637; Moenkhausia pyrophthalma MPEG 2837; Moenkhausia sp. MPEG 2469; Paragoniates alburnus MPEG 2704; Phenacogaster sp. MPEG 1947; Poptella sp. MPEG 9039; Pristella maxillaris MPEG 7614, MPEG 7620, MPEG 7621, MPEG 7616; Pristobrycon aureus MPEG 1506; Pristobrycon calmoni MPEG 2753; Pygocentrus nattereri MPEG 1541, MPEG 2371, MPEG 2492, MPEG 2476, MPEG 2493, MPEG 2439, MPEG 2484, MPEG 2568, MPEG 2443, MPEG 2471, MPEG 2491, MPEG 2721, MPEG 2485, MPEG 2486, MPEG 2572, MPEG 2573, MPEG 2574, MPEG 2575, MPEG 2576, MPEG 2577 , MPEG 2578, MPEG 2579, MPEG 2622, MPEG 2752, MPEG 2756 , MPEG 2775, MPEG 2909, MPEG 2849, MPEG 2850, MPEG 2851 MPEG 4827, UF 44925, UF 44944, UF 44953; Roeboides myersii MPEG 2392, MPEG 2498, MPEG 2499, MPEG 2763, MPEG 6425 MPEG 2602, MPEG 2610; Roeboides sp. MPEG 2900, MPEG 1875, MPEG 1873, MPEG 1240, MPEG 2394, MPEG 2461, MPEG
2500, MPEG 2703, MPEG 2706, MPEG 2724, MPEG 2640, MPEG 2645, MPEG 2628, MPEG 2899, MPEG 2901, MPEG 2840, MPEG 2841, MPEG 2842, MPEG 2936, MPEG 9030, MPEG 9035, MPEG 9036, MPEG 9037, UF 44936; Serrasalmus elongatus MPEG 6430; Serrasalmus rhombeus MPEG 2372, MPEG 2488, MPEG 5429, MPEG 2722, MPEG 2489, MPEG 2474, MPEG 2754, MPEG 2771, MPEG 2942, UF 44943, UF 44952; Serrasalmus sp. MPEG 2623, MPEG 1505, MPEG 1539, MPEG 2373, MPEG 2473, MPEG 2638, MPEG 2490, MPEG 2755, MPEG 2774, MPEG 2852, MPEG 2853, MPEG 2854, MPEG 2855, MPEG 6428, MPEG 6429, UF 44939; Triportheus aff. angulatus MPEG 9025; Triportheus albus MPEG 2903, MPEG 7970, MPEG 7983, MPEG 1735, MPEG 1727; Triportheus angulatus MPEG 2395, MPEG 2764, MPEG 2902, MPEG 2905, MPEG 2937, MPEG 6400, MPEG 6427, MPEG 6426, MPEG 7947, UF 44935; Triportheus elongatus MPEG 2761, MPEG 9026; Triportheus sp. MPEG 2904, MPEG 1728, MPEG 2450, MPEG 2454, MPEG 2451, MPEG 2441, MPEG 2715, MPEG 2503, MPEG 2601, MPEG 2641, MPEG 2750, MPEG 2627, MPEG 2765, MPEG 2843; CRENUCHIDAE: Crenuchus spilurus MPEG 1978, MPEG 7608; Microcharacidium sp. MPEG 7615; CTENOLUCIIDAE: Boulengerella lucius MPEG 7553; CURIMATIDAE: Curimata inornata MPEG 7570, MPEG 7974, MPEG 8005; Curimata sp.1 MPEG 2821; Curimata sp.2 MPEG 9034, Curimatopsis sp. MPEG 7619, MPEG 2822, MPEG 7957; ERYTHRINIDAE: Erythrinus erythrinus MPEG 2908, MPEG 2938, MPEG 9023, MPEG 9050; Hoplerythrinus unitaeniatus MPEG 4845, MPEG 4689, MPEG 7535, MPEG 7546; Hoplias malabaricus MPEG 1820, MPEG 2362, MPEG 2464, MPEG 2544, MPEG 2718, MPEG 2545, MPEG 2580, MPEG 7918, MPEG 7919, MPEG 7920, MPEG 7921, MPEG, 7923, MPEG 7924, MPEG 7925, MPEG 7926, MPEG 7927, MPEG 7928, MPEG 7929, MPEG 7929, MPEG 7930, MPEG 7930, MPEG 7922, MPEG 2776, MPEG 2907, MPEG 2839, MPEG 2939, MPEG 4840, MPEG 6403, MPEG 7536, MPEG 9022, MPEG 7956, MPEG 7985, MPEG 7990; Hoplias sp. MPEG 1819, MPEG 2748, MPEG 4836; GASTEROPELECIDAE: Carnegiella sp. MPEG 9024, MPEG 9032; Carnegiella strigata MPEG 9042, MPEG 9043; Gasteropelecus sternicla MPEG 4446, MPEG 7593, MPEG 7596, MPEG 9040, MPEG 9041, MPEG 9049; Thoracocharax stellatus MPEG 8020, MPEG 8040; HEMIODONTIDAE: Hemiodus unimaculatus MPEG 4676, MPEG 7547, MPEG 7548, MPEG 7549, MPEG 7550, MPEG 7551, MPEG 7951, MPEG 8010, MPEG 8028; LEBIASINIDAE: Copella arnoldi MPEG 2941; Copella nattereri MPEG 2940 Nannostomus eques MPEG 7601, MPEG 7625; Nannostomus nittidus MPEG 2823; Pyrrhulina filamentosa MPEG 7626, MPEG 7627, MPEG 9028.

Clupeiformes: ENGRAULIDIDAE: Anchovia sp. MPEG 7635 , MPEG 7631; Anchovia surinamensis MPEG 9063, MPEG 9065, MPEG 9066; Lycengraulis batesii MPEG 1328, MPEG 6320, MPEG 2820; Lycengraulis sp. MPEG 2719, MPEG 2819; Pterengraulis atherinoides MPEG 6308, MPEG 8035; PRISTIGASTERIDAE: Pellona castelnaeana MPEG 8002, MPEG 8034; Pellona flavipinnis MPEG 2639, MPEG 2637; Pellona sp. MPEG 2483, MPEG 2818, MPEG 7617.

Cyprinodontiformes: ANABLEPIDAE: Anableps microlepis MPEG 1260, MPEG 1258, MPEG 1254, MPEG 1256, MPEG 1255, MPEG 1253, MPEG 1257; POECILIIDAE: Pamphorichthys sp. MPEG 7613; Poecilia sp.1 MPEG 7612, MPEG 7958; Poecilia sp.2 MPEG 7960; Poecilia sp.3 MPEG 7961; Poecilia sp.4 MPEG 7962; Poecilia sp.5 MPEG 7964; RIVULIDAE: Rivulus sp. MPEG 7610, MPEG 7611, MPEG 7632, MPEG 7959.

Gymnotiformes: APTERONOTIDAE: Adontosternarchus sp. MPEG 1154, MPEG 1155, MPEG 1153, MPEG 2390, MPEG 2427, MPEG 2435, MPEG 2463, MPEG 2606, MPEG 6413; 
Apteronotus albifrons MPEG 1466, MPEG 1150, MPEG 1152, MPEG 2369, MPEG 2513, MPEG 2434, MPEG 2514, MPEG 3707, MPEG 7973, MPEG 1160; Apteronotus sp. MPEG 2516, MPEG 2515, MPEG 3715, MPEG 1162, MPEG 1158, MPEG 1161, MPEG 1159, MPEG 2393, MPEG 2433, MPEG 5705, MPEG 2517, MPEG 2518, MPEG 4680; Sternarchella cf. terminalis MPEG 7989; Sternarchella sp. MPEG 1163, MPEG 2808, MPEG 2809; Sternarchogiton nattereri MPEG 2804, MPEG 2805, MPEG 2806, MPEG 3710; Sternarchogiton porcinum MPEG 3698, MPEG 3696, MPEG 3697; Sternarchogiton sp. MPEG 3705, MPEG 2807, MPEG 2811; Sternarchorhamphus muelleri MPEG 3701, MPEG 2929, MPEG 3703, MPEG 3711, MPEG 3712, MPEG 3700; Sternarchorhamphus sp. MPEG 3718, MPEG 2812, MPEG 2813, MPEG 2814, MPEG 3713; GYMNOTIDAE: Electrophorus electricus MPEG 2243, MPEG 2244, MPEG 2245, MPEG 2482, MPEG 2455, MPEG 4022, MPEG 3818, MPEG 4021, MPEG 2799, MPEG 959, MPEG 2975, MPEG 2978, MPEG 4019, MPEG 2976, MPEG 2977 , MPEG 3818, UF 44939; Gymnotus carapo MPEG 1166, MPEG 2519, MPEG 2520, MPEG 2431, MPEG 5463, MPEG 2886, MPEG 2928, MPEG 4017, MPEG 7563, MPEG 7539, MPEG 9072, MPEG 9073, MPEG 9075; Gymnotus sp. MPEG 2365, MPEG 2521, MPEG 6431, MPEG 7992; HYPOPOMIDAE: Brachyhypopomus brevirostris MPEG 7597; Brachyhypopomus pinnicaudatus MPEG 2797, MPEG 2816; Brachyhypopomus sp. MPEG 7588, MPEG 2731; Hypopomus sp. MPEG 1129, MPEG 1132, MPEG 1133, MPEG 1130, MPEG 2437, MPEG 3732, MPEG 9009, MPEG 9076, MPEG 9080, MPEG 9082; Hypopygus lepturus MPEG 1100, MPEG 1093, MPEG 2423, MPEG 2436, MPEG 2714, MPEG 2522, MPEG 2605, MPEG 2815; Hypopygus sp. MPEG 7600, MPEG 9078; Microsternarchus bilineatus MPEG 8000; Steatogenys elegans MPEG 1139, MPEG 1137, MPEG 7999, MPEG 8017, MPEG 8043; Steatogenys sp. MPEG 9081; RHAMPHICHTHYIDAE: Rhamphichthys marmoratus MPEG 7540; Rhamphichthys rostratus MPEG 2798, MPEG 4681, MPEG 4684; Rhamphichthys sp. MPEG 1157, MPEG 1156, MPEG 2370, MPEG 2523, MPEG 2569, MPEG 2524, MPEG 3036, MPEG 2727, MPEG 2885; STERNOPYGIDAE: Distocyclus conirostris MPEG 2447; Eigenmannia humboldtii MPEG 1091, MPEG 1077, MPEG 2509, MPEG 2425, MPEG 2430, MPEG 2510, MPEG 2810; Eigenmannia sp.1 MPEG 6434, MPEG 6437, MPEG 6435, MPEG 6436, MPEG 2800, MPEG 1065, MPEG 1067, MPEG 1075, MPEG 1063, MPEG 1064, MPEG 1068, MPEG 1072, MPEG 1073, MPEG 1061, MPEG 1062, MPEG 1070, MPEG 1074, MPEG 1081, MPEG 1069, MPEG 1071, MPEG 1082, MPEG 1076, MPEG 1066, MPEG 2387, MPEG 2388, MPEG 2460, MPEG 2424, MPEG 2428, MPEG 2429, MPEG 2462, MPEG 2438, MPEG 2445, MPEG 2720, MPEG 2507, MPEG 2508, MPEG 2571, MPEG 2598, MPEG 2732, MPEG 2733, MPEG 2734, MPEG 2888, MPEG 2890, MPEG 2891, MPEG 2892, MPEG 2889, MPEG 2930, MPEG 2931; Eigenmannia sp.2 MPEG 9077, MPEG 9079; Rhabdolichops sp. MPEG 1173, MPEG 1174, MPEG 1175, MPEG 1176, MPEG 1190, MPEG 2389, MPEG 2446, MPEG 2803; Rhabdolichops troscheli MPEG 1189, MPEG 1169, MPEG 2604, MPEG 3702, MPEG 2801, MPEG 2802; Sternopygus macrurus MPEG 1085, MPEG 1084, MPEG 1089, MPEG 1083, MPEG 1087, MPEG 1088, MPEG 2364, MPEG 2391, MPEG 2511, MPEG 2426 , MPEG 2432, MPEG 2459, MPEG 2512, MPEG 5468, MPEG 3704, MPEG 2887, MPEG 2932, MPEG 3708, MPEG 4014, MPEG 4690, MPEG 4497, MPEG 6433, MPEG 6432, MPEG 7585, MPEG 7537; Sternopygus sp. MPEG 9074.

Lepidosireniformes: LEPIDOSIRENIDAE: Lepidosiren paradoxa MPEG 2980.

Osteoglossiformes: ARAPAIMIDAE: Arapaima gigas MPEG 2099; : Osteoglossum bicirrhosum MPEG 1057, MPEG 2554, MPEG 2555, MPEG 2556, UF 44934.
Perciformes: CENTROPOMIDAE: Centropomus sp. MPEG 2259; CICHLIDAE: Acaronia nassa MPEG 7576, MPEG 7577, MPEG 2860, MPEG 2943; Aequidens pallidus MPEG 7533, MPEG 2944, MPEG 3834; Aequidens sp. MPEG 9054, MPEG 7594; Apistogramma luelingi MPEG 7590, MPEG 7607; Apistogramma sp. MPEG 7589, MPEG 7598, MPEG 2857, MPEG 2945, MPEG 7595, MPEG 7599, NRM 19187; Astronotus ocellatus MPEG 1032, MPEG 2496, MPEG 2497; Chaetobranchopsis orbicularis MPEG 6405; Chaetobranchopsis sp. MPEG 2778, MPEG 2895, MPEG 2859; Chaetobranchus sp. MPEG 2893, MPEG 2894; Cichla melaniae MPEG 7541; Cichla monoculus MPEG 2363, MPEG 7561, MPEG 2101; Cichla nigromaculata MPEG 7562; Cichla ocellaris MPEG 2494; Cichla sp.1 MPEG 7945, MPEG 7945, MPEG 8029; Cichla sp.2 MPEG 7946; Cichla temensis MPEG 4607, MPEG 7544; Cichlasoma sp. MPEG 2374, MPEG 2375, MPEG 2495; Crenicichla cincta MPEG 9055; Crenicichla macrophthalma MPEG 7582, MPEG 8031; Crenicichla notophthalmus MPEG 7633; Crenicichla regani MPEG 2861, MPEG 7994; Crenicichla reticulata MPEG 7571; Crenicichla sp.1 MPEG 1600, MPEG 1589, MPEG 2449, MPEG 2779, MPEG 2946, MPEG 3831, MPEG 7573, MPEG 7575, MPEG 7580, MPEG 7587, MPEG 7705, MPEG 7579, MPEG 7581, MPEG 7583, MPEG 7634, MPEG 8021, MPEG 8039; Crenicichla sp.2 MPEG 9056; Crenicichla sp.3 MPEG 8032; Crenicichla strigata MPEG 7574, MPEG 7572; Geophagus camopiensis MPEG 4605; Geophagus sp. MPEG 2777, MPEG 2858, MPEG 7996; Geophagus surinamensis MPEG 7530, MPEG 7531, MPEG 7543, MPEG 7954; Satanoperca jurupari MPEG 7532, MPEG 7534, MPEG 7542, MPEG 7948, MPEG 7948, MPEG 7953, MPEG 7987, MPEG 7991, MPEG 8008, MPEG 8014, MPEG 8015, MPEG 8019, MPEG 8036, MPEG 8042; GOBIIDAE: Awaous flavus MPEG 1223; Bathygobius sp. MPEG 1224; Eleotris sp. MPEG 7604; Evorthodus sp. MPEG 7606; Gobioides sp. MPEG 9069, MPEG 9070; Gobionellus sp. MPEG 7609; MUGILIDAE: Mugil curema MPEG 1225, MPEG 9068; POLYCENTRIDAE: Monocirrhus polyacanthus MPEG 9057; Polycentrus schomburgkii MPEG 7578; SCIAENIDAE: Cynoscion microlepidotus MPEG 2316; Cynoscion sp. MPEG 2315; Macrodon ancylodon MPEG 1231; Pachypops fourcroi MPEG 7566; Pachypops sp. MPEG 9064, MPEG 9067; Pachypops trifilis MPEG 7980, MPEG 8006; Plagioscion auratus MPEG 1239, Plagioscion sp. MPEG 2313, MPEG 2308, MPEG 2310, MPEG 2314, MPEG 2562; Plagioscion squamosissimus MPEG 2309, MPEG 2311, MPEG 2564, MPEG 2947, MPEG 7564, MPEG 7565, MPEG 7567, MPEG 7976, MPEG 7981; Plagioscion surinamensis MPEG 3594, MPEG 2588, MPEG 2616, MPEG 1983, MPEG 1673, MPEG 2565, MPEG 2563, MPEG 2589, MPEG 2617, MPEG 2749, MPEG 2629, MPEG 2795.

Pleuronectiformes: ACHIRIDAE: Achirus achirus MPEG 7557, MPEG 7963; Achirus sp. MPEG 9052; PARALICHTHYIDAE: Paralichthys brasiliensis MPEG 7605; Paralichthys sp. MPEG 7949.

Rajiformes: POTAMOTRYGONIDAE: Potamotrygon motoro MPEG 2466, MPEG 2468; Potamotrygon orbignyi MPEG 1332, MPEG 1331, MPEG 1809, MPEG 2475, MPEG 7591; Potamotrygon scobina MPEG 7592; Potamotrygon sp. MPEG 2480, MPEG 2481, MPEG 2479, MPEG 2457.

Siluriformes: ASPREDINIDAE: Aspredinichthys filamentosus MPEG 1334; Aspredo aspredo MPEG 1340, MPEG 4379, MPEG 4380; Bunocephalus aleuropsis MPEG 9001, MPEG 9002; AUCHENIPTERIDAE: Ageneiosus inermis MPEG 1454, MPEG 2593, MPEG 2916, MPEG 2525, MPEG 2526, MPEG 2527, MPEG 2528, UF 44937; Ageneiosus sp.1 MPEG 2949; Ageneiosus sp.2 MPEG 2386, MPEG 2612, MPEG 2796, MPEG 2873 , MPEG 7569; Ageneiosus ucayalensis MPEG 2478, MPEG 2594, 
MPEG 2760, MPEG 2747, MPEG 2698, MPEG 2948, MPEG 7568, MPEG 7975, MPEG 7979; Asterophysus sp. MPEG 6407; Auchenipterichthys longimanus MPEG 9012; Auchenipterus nuchalis MPEG 1985, MPEG 2440, UF 44927, MPEG 2448, MPEG 2789, MPEG 2910, MPEG 2874, MPEG 2950, MPEG 6408, MPEG 6406; Centromochlus heckelii MPEG 9016, MPEG 9017, MPEG 9046; Pseudauchenipterus nodosus MPEG 1293, MPEG 3751; Tatia intermedia MPEG 2875, MPEG 4350; Trachelyopterus galeatus MPEG 1447, MPEG 1433, MPEG 2537, MPEG 2624, MPEG 2538 , MPEG 2540, MPEG 2584, MPEG 2770, MPEG 2619, MPEG 2620 , MPEG 2744, MPEG 2792, MPEG 2921, MPEG 4443, MPEG 7603, MPEG 7978, MPEG 8012, MPEG 8037, MPEG 2923; Trachelyopterus sp. MPEG 1282, MPEG 2380, MPEG 2381, MPEG 2701, MPEG 2487, MPEG 2472, MPEG 2539, MPEG 2536, MPEG 2444, MPEG 2570, MPEG 2581, MPEG 2582, MPEG 2583, MPEG 2585, MPEG 2586, MPEG 2609, MPEG 2618, MPEG 2743, MPEG 2741, MPEG 2742, MPEG 2790, MPEG 2791, MPEG 2699, MPEG 2922, MPEG 2924, MPEG 2925, MPEG 2926, MPEG 2927, MPEG 2876, MPEG 2877, MPEG 2878, MPEG 2879, MPEG 2880, MPEG 2881, MPEG 2882, MPEG 2883, MPEG2884, MPEG 2951, MPEG 2952, MPEG 2953, MPEG 4372, MPEG 6402; CALLICHTHYIDAE: Callichthys callichthys MPEG 2866; Hoplosternum littorale MPEG 2379, MPEG 2587, MPEG 2696, MPEG 2700, MPEG 2867, MPEG 2954, MPEG 4493, MPEG 6404, MPEG 2504, MPEG 2505, MPEG 4167, MPEG 7602, Megalechis personata MPEG 2955; Megalechis thoracata MPEG 2868, MPEG 2506, MPEG 2702, MPEG 2869, MPEG 9003, MPEG 9004, MPEG 9005. MPEG 9006, MPEG 9007; CETOPSIDAE: Cetopsis sp. MPEG 8026; Hemicetopsis sp. MPEG 8027; DORADIDAE: Acanthodoras sp. MPEG 2956; Anadoras sp. MPEG 1437, MPEG 1438, MPEG 2382, MPEG 2541, MPEG 2442, MPEG 2465, MPEG 2542, MPEG 2543, MPEG 2726, MPEG 2613, MPEG 2729, MPEG 2746, MPEG 2745, MPEG 2632, MPEG 2793, MPEG 2920, MPEG 2863, MPEG 2957, MPEG 6417, MPEG 6419; Doras eigenmanni MPEG 6422, MPEG 6415, MPEG 6423, MPEG 6420; Doras sp. MPEG 2794, MPEG 2919, MPEG 2864, MPEG 2865, MPEG 2958; Hassar sp. UF 44938; Lithodoras dorsalis MPEG 1281; HEPTAPTERIDAE: Gladioglanis machadoi MPEG 7998;; Pimelodella altipinnis MPEG 8004, NRM 15467; Pimelodella cristata MPEG 1238, MPEG 1434, MPEG 1442, MPEG 2456, MPEG 2458, MPEG 2559, MPEG 2595 , MPEG 2600, MPEG 2736, MPEG 2631, MPEG 2783, MPEG 2862, MPEG 2968, MPEG 2969, MPEG 6418, MPEG 7950, MPEG
7950, MPEG 8038; Pimelodella gracilis MPEG 7988, MPEG 9020; Pimelodella sp. MPEG 1440; Rhamdia quelen MPEG 1439, MPEG 1786, MPEG 2385, MPEG 2560, MPEG 2621, MPEG 2737, MPEG 2782, MPEG 2911, MPEG 2912, MPEG 2913, MPEG 2972, MPEG 4447, MPEG 4448, NRM 15474; Rhamdia sp. MPEG 9013, MPEG 9019; Incertae sedis: Phreatobius cisternarum MZUSP 88396, MZUSP 85475; LORICARIIDAE: Ancistrus sp.1 MPEG 2712, Ancistrus sp.2 MPEG 6410, MPEG 7643, MPEG 7644, Chaetostoma sp. MPEG 7642, MPEG 7645; Farlowella aff. knerii MPEG 9008; Farlowella amazona MPEG 2599, MPEG 2725; Farlowella sp. MPEG 2713; Hemiancistrus sp. MPEG 7986, Hemiodontichthys acipenserinus MPEG 6409; Hemiodontichthys sp. MPEG 2711, MPEG 2786, MPEG 2870, MPEG 2959; Hypoptopoma sp. MPEG 2738; Hypostomus sp. MPEG 2709, MPEG 2549, MPEG 2550, MPEG 2552, MPEG 2596, MPEG 2739, MPEG 2965, MPEG 2966, MPEG 9010, MPEG 9011; Lasiancistrus sp. MPEG 2967; Limatulichthys sp. MPEG 2962; Liposarcus pardalis MPEG 2546, MPEG 2551, MPEG 2547, MPEG 2705; Loricaria catphracta MPEG 1485, MPEG 2376, MPEG 2548, MPEG 2553, MPEG 2707, MPEG 2708, MPEG 2597, MPEG 2615, MPEG 2740, MPEG 2788, MPEG 2872, MPEG 2960, MPEG 2961, Panaque sp. MPEG 1490; Pseudoloricaria sp. MPEG 2633, MPEG 2787, MPEG 2917, MPEG 2918, MPEG 2871, MPEG 9014, MPEG 9015, MPEG 7967, MPEG 8007; Pterygoplichthys sp. MPEG 2728, MPEG 2780; Reganella sp. MPEG 2963, MPEG 2964; PIMELODIDAE: Brachyplatystoma vaillantii MPEG 1271; Hypophthalmus marginatus MPEG 4679; Pimelodus blochii MPEG 2383, MPEG 2561, MPEG 2784, MPEG 2914, MPEG 3639, MPEG 6416, MPEG 7977, MPEG 1448, MPEG 1449, MPEG 2477, MPEG 2611, MPEG 2730, MPEG 2735, MPEG 2630; Pimelodus ornatus MPEG 2557, MPEG 2558, MPEG 2785, MPEG 2915, MPEG 2971, MPEG 8033; Pimelodus sp.1 MPEG 2384, MPEG 2453, MPEG 2970, MPEG 9021; Pimelodus sp.2 MPEG 9085; Pinirampus pirinampu MPEG 8009; Zungaro zungaro MPEG 1270, MPEG 1273, MPEG 8001; PSEUDOPIMELODIDAE: Batrochoglanis raninus MPEG 2710, MPEG 7641; TRICHOMYCTERIDAE: Henonemus taxistigmus MPEG 9018.

S y n b r a n c h if or m e s: S Y N B R A N C H I D A E : Synbranchus lampreia MZUSP 50184; Synbranchus marmoratus MPEG 3381, MPEG 9053, MPEG 8023, MPEG 8041; Synbranchus sp. MPEG 2098, MPEG 2979, MPEG 3865, MPEG 3374, MPEG 3377.

Tetraodontiformes: TETRAODONTIDAE: Colomesus asellus MPEG7558,MPEG7559,MPEG9031,MPEG7955;Colomesuspsittacus MPEG 1216, MPEG 1215, MPEG 1217, MPEG 7560. 